Similarly to the applications described in the first part of this publication, positron emission tomography with computed tomography (PET/CT) is also gaining importance in monitoring a tumour's response to therapy and diagnosing breast cancer recurrences. This is additionally caused by the fact that many new techniques (dual-time point imaging, positron emission tomography with magnetic resonance PET/MR, PET/ CT mammography) and radiotracers (16 $\alpha$-18F-fluoro-17 $\beta$-estradiol, 18F-fluorothymidine) are under investigation. The highest sensitivity and specificity when monitoring response to treatment is achieved when the PET/CT scan is made after one or two chemotherapy courses. Response to anti-hormonal treatment can also be monitored, also when new radiotracers, such as FES, are used. When monitoring breast cancer recurrences during follow-up, PET/CT has higher sensitivity than conventional imaging modalities, making it possible to monitor the whole body simultaneously. New techniques and radiotracers enhance the sensitivity and specificity of PET and this is why, despite relatively high costs, it might become more widespread in monitoring response to treatment and breast cancer recurrences.

Key words: breast cancer, positron emission tomography, computed tomography, radiopharmaceuticals.

Contemp Oncol (Pozn) 2016; 20 (3): 205-209 DOI: $10.5114 /$ wo.2016.61560

\section{The application of positron emission tomography (PET/CT) in diagnosis of breast cancer. Part II. Diagnosis after treatment initiation, future perspectives}

\author{
Elżbieta Jodłowska ${ }^{1}$, Rafał Czepczyński ${ }^{1}$, Agata Czarnywojtek ${ }^{1}$, \\ Amanda Rewers ${ }^{1}$, Grażyna Jarząbek ${ }^{2}$, Witold Kędziaa ${ }^{2}$, Marek Ruchała ${ }^{1}$
}

${ }^{1}$ Department of Endocrinology, Poznan University of Medical Sciences, Poznan, Poland ${ }^{2}$ Department of Gynecology, Poznan University of Medical Sciences, Poznan, Poland

\section{Response to treatment}

Positron emission tomography with computed tomography (PET/CT) with ${ }^{18} \mathrm{~F}$-fluorodeoxyglucose (FDG) tracer is gaining importance in oncology as a method of assessing response to treatment. For a variety of malignant diseases (such as lymphoma, lung, or oesophageal cancer) the reduction or normalisation of ${ }^{18} \mathrm{~F}$-FDG uptake by the tumour after several chemotherapy courses has been reported to correlate with the response to treatment, as well as with the recurrence rate and overall survival [1-3]. Can similar relationships be found for breast cancer patients? Would a PET/CT scan between courses of neoadjuvant chemotherapy affect the course of treatment? The answer is still not firmly established but is widely researched due to its significance, particularly for patients with metastatic breast cancer. Figure 1 shows the PET/CT scan of a patient with breast cancer in the left breast, who also had metastases in axillary lymph nodes and left iliac bone. How can the response to treatment be monitored among such patients?

In recent years there have been a considerable number of publications regarding this subject. They were gathered in three meta-analyses concerning $\mathrm{PET}$ and PET/CT as methods that allow the response to neoadjuvant chemotherapy to be monitored [4-6]. Table 1 shows the results of the three meta-analyses. Whereas both PET and PET/CT have a high sensitivity, their insufficient average specificity (approximately $70 \%$ ) renders it impossible to make them a standard method to monitor response to neoadjuvant chemotherapy. Nonetheless, specificity in separate original research papers varies considerably; for instance, among the publications gathered in the meta-analysis of Cheng et al. the specificity varied from $30 \%$ to $96 \%$. It is primarily a result of heterogeneous inclusion criteria to each study, particularly as far as histologic tumour subtypes and tumour receptor expression are concerned [4].

Furthermore, the separate publications are also different in terms of the applied protocol of PET/CT examination. According to the meta-analysis of Wang et al., the most appropriate time point to conduct PET/CT is after the first or second course of chemotherapy because then it has the greatest impact on treatment [6]. The comparison of sensitivity and specificity after one and two chemotherapy courses showed no significant difference between the two - sensitivity and specificity were equal to $74 \%$ and $86 \%$, respectively, after the first course and $77 \%$ and $84 \%$, respectively, after the second course of chemotherapy [5]. 

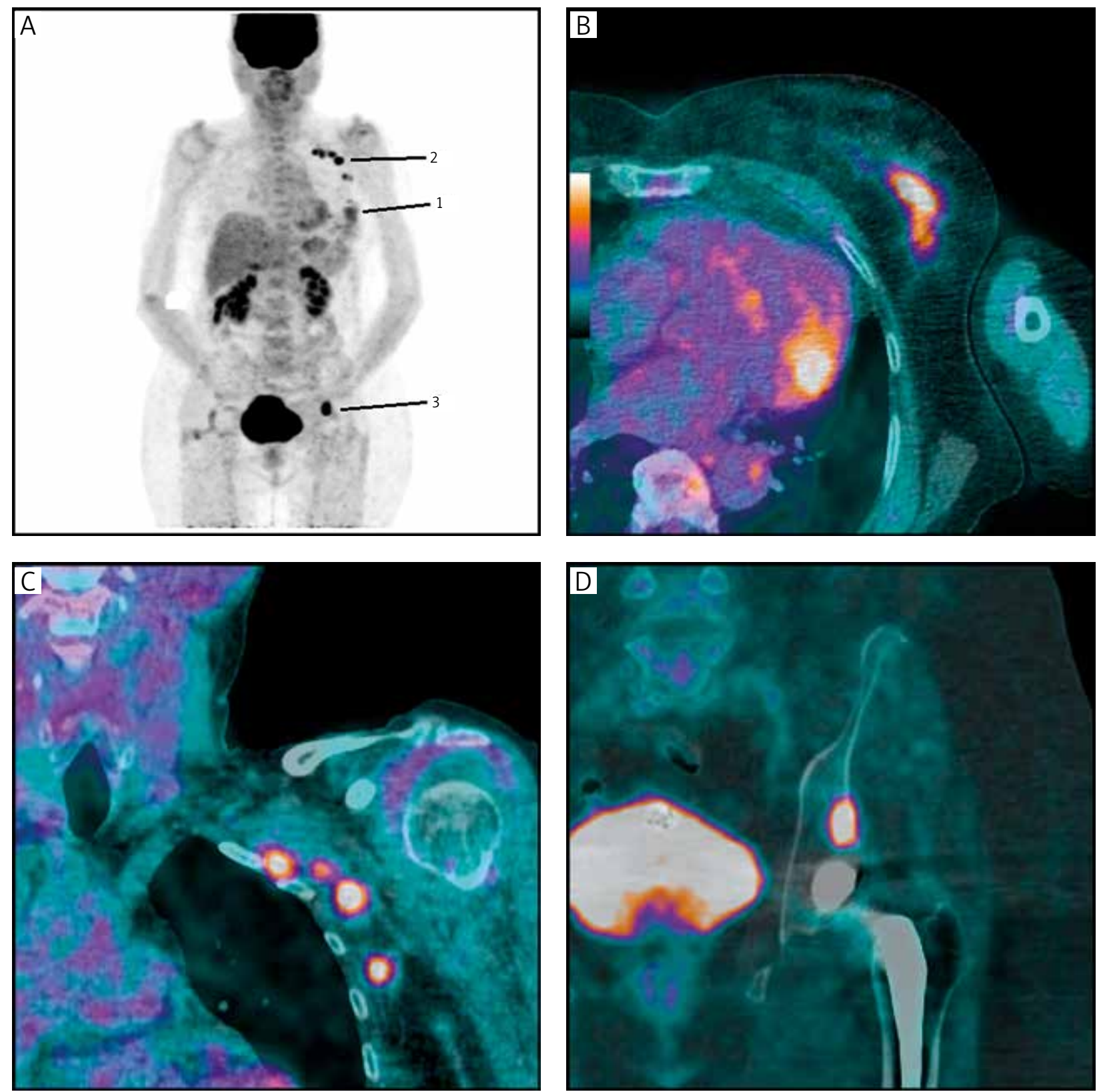

Fig. 1. PET with ${ }^{18}$ F-FDG in a 53 y.o. patient with cancer of the left breast (1), axillary lymph node metastases (2) and iliac bone metastasis (3; A). Below PET/CT fusion images of these foci (B-D)

Another challenging issue is the choice of an appropriate SUV $V_{\max }$ cut-off value, which would indicate response to treatment. It is difficult not only due to the heterogeneity of breast cancer tumours, but also because of the unstandardised histopathological criteria. A change of cut-off value of SUV $V_{\max }$ between $-55 \%$ and $-65 \%$ after the second course of chemotherapy has been suggested [5]. Then, a decrease in ${ }^{18} \mathrm{~F}$-FDG uptake by the breast tumour lower than 55\% would allow early identification of tumours non-sensitive to chemotherapy, which usually do not respond to further courses of such treatment [5]. The study of Kolesnikov-Gauthier et al. reported an SUV ${ }_{\max }$ decline of

Table 1. PET and PET/CT as methods of monitoring response to neoadjuvant chemotherapy - comparison of meta-analyses

\begin{tabular}{|c|c|c|c|c|}
\hline Reference & $\begin{array}{c}\text { Number of studies included } \\
\text { in the meta-analysis }\end{array}$ & $\begin{array}{c}\text { Sensitivity of }{ }^{18} \mathrm{~F}-\mathrm{FDG} \\
\text { PET }\end{array}$ & $\begin{array}{l}\text { Specificity of } \\
{ }^{18} \text { F-FDG PET }\end{array}$ & $\begin{array}{l}\text { Number of chemotherapy } \\
\text { courses at the time of the scan }\end{array}$ \\
\hline Cheng et al., 2012 & 17 & $84 \%$ & $71 \%$ & no data \\
\hline Mghanga et al., 2013 & 15 & $81 \%$ & $79 \%$ & $1-4$ \\
\hline Wang et al., 2012 & 16 & $84 \%$ & $66 \%$ & $1-8$ \\
\hline
\end{tabular}


less than $15 \%$ after the first course of chemotherapy to be strongly correlated with lack of response to treatment [7].

\section{Receptor expression and monitoring response to treatment}

Sensitivity and specificity of PET and PET/CT in monitoring response to treatment depends on the receptor expression: those for oestrogen (ER), progesterone (PgR), and human epidermal growth factor 2 (HER2). It has been suggested that PET is of particular utility for HER2- tumours $[8,9]$. The study of Koolen et al. confirms that the decline in ${ }^{18} \mathrm{~F}$-FDG uptake is correlated with response to neoadjuvant chemotherapy for HER2- tumours (including triple negative and ER+ breast cancer), but not for HER2+ tumours [8]. Moreover, monitoring the pace of glycolysis in $\mathrm{PET} / \mathrm{CT}$ for ER+ HER2- breast cancer patients allows to distinguish tumours sensitive and resistant to chemotherapy with an $89 \%$ sensitivity [9]. For a group of patients with similar tumours, Zucchini et al. achieved a 100\% sensitivity, but only a $38 \%$ specificity [10]. What is more, for triple negative breast cancer it was possible not only to identify the tumours resistant to chemotherapy, but also to estimate the disease-free survival (DFS), by measuring the SUV $_{\max }$ change after chemotherapy [11]. These differences, dependent on tumour receptor expression, show the reasons for the above described discrepancies between the obtained specificities in different studies that monitor response to chemotherapy with PET or PET/CT.

There have also been a number of studies that assess the potential role of PET/CT in monitoring response to anti-hormonal treatment. Among such patients, the decline in ${ }^{18} \mathrm{~F}$-FDG uptake has been found to correlate with progression-free survival (PFS) [12]. For treatment with aromatase inhibitors there was a correlation between the change in SUV $_{\max }$ and the proliferation of the tumour after therapy [13].

What is more, PET/CT allows us to assess the heterogeneity of the response to treatment within both the primary tumour and metastases. Among $48 \%$ of patients with dominating bone metastases a varying response to treatment was reported in the study by Huyge et al. [14]. Those patients had a significantly lower time to progression (TTP).

As already described in the first part of this publication, PET has a high specificity in the detection of lymph node metastases. Thus, PET/CT is an effective method in the assessment of response to therapy of lymph node metastases among stage II and III patients. According to a meta-analysis by Wang et al., it has a sensitivity of $92 \%$ but varying specificity [6]. Statistically significant differences between responders and non-responders to chemotherapy were visible after the first course of treatment.

\section{Detection of disease progression and recurrence}

$\mathrm{PET} / \mathrm{CT}$ is widely applied in monitoring breast cancer progression and recurrence. The sensitivity of PET/CT in recurrence detection is higher than that of CT and the CA 15-3 marker [15]. According to the study by Murakami et al., PET/ CT had a $96 \%$ sensitivity and $91 \%$ specificity, and thus high negative and high positive predictive values (92\% and 95\%, respectively). These values were significantly higher than for PET alone (80\%, 82\%, 83\%, and $78 \%$, respectively) [16]. In other studies, the sensitivity of PET/CT was 93-97\%, and its specificity was $92-100 \%[15,17]$. Furthermore, it was also shown that a PET/CT scan conducted to assess breast cancer recurrence results in a change of treatment among 20-48\% of patients $[15,17]$. A more accurate localisation of the tumour and an increased resolution in imaging the axillary tail of Spence, skin, and thoracic walls can be achieved by scanning breasts and axillae in a prone position [18]. Nonetheless, it was not proven that such an examination improves the accuracy of $\mathrm{N}$ assessment in the TNM system [18].

In the case of a suspected recurrence due to the elevation of CEA and/or CA 15-3, PET/CT has a higher sensitivity (95\% vs. 33\%) and accuracy (94\% vs. $48 \%$ ) than conventional imaging. In the examined group, PET/CT resulted in a change of treatment protocol among $54 \%$ of patients. However, it has been stressed by the authors that PET/CT scan is indicated only in the case of a constantly increasing concentration of the marker [19].

\section{New methods}

\section{Positron emission mammography}

PET scanners dedicated to the imaging of breasts have been developed to improve the detection of small breast tumours. This technique has been named positron emission mammography (PEM). Thanks to its enhanced spatial resolution, PEM has a higher sensitivity in detection of lesions smaller than $2 \mathrm{~cm}$ in diameter [20]. Nevertheless, it does not allow scanning of the whole body during a single examination. There are different types of PEM: O scanner (examination in a supine position) and C scanner (examination in a prone position) [20].

\section{Dual-time point imaging}

Dual-time point imaging (usually 60 and 90-360 minutes after the administration of a radioactive tracer), connected with calculating the difference between the two SUV $_{\max }$ measurements, allows to distinguish between benign and malignant lesions, both in primary diagnosis and in cases of suspicion of breast cancer recurrence [21, 22]. Furthermore, this method also increases the sensitivity for tumours with a low diameter, tumours in breasts with a high density, and in discerning invasive and non-invasive breast cancer [21]. As a rule, malignant tumours tend to have an increasing uptake of tracer, whereas both healthy tissues and benign lesions present a decline in ${ }^{18} \mathrm{~F}-\mathrm{FDG}$ uptake within a tested time frame [21]. Such an examination is not sensitive enough to become a primary diagnostic method for all breast cancer patients; however, it gives a significant increase in sensitivity when detecting small breast tumours [21]. Dual-time point imaging does not enhance the assessment of lymph nodes' involvement: it has no effect on diagnostic accuracy and can increase the percentage of false positive results [23].

\section{Positron emission tomography/magnetic resonance}

Magnetic resonance (MR) is a method that is applied in breast tumour imaging. Therefore, a fusion of PET and MR 
images might be extremely useful in the management of oncology patients. There are an increasing number of PET/ MR scanners throughout the world. The fusion of PET with $M R$ in primary breast cancer detection has resulted in an increase in positive predictive value from $77 \%$ for $M R I$ to $98 \%$, and specificity from $53 \%$ to $97 \%$ [24]. PET/MR also facilitates the choice of lesions that should be subjected to biopsy.

Studies that monitored response to treatment yielded similar results, where the PET image reflected the tumour metabolism, and the MR showed the lesion's vasculature. The study of Partridge et al. showed that certain parameters in both techniques are correlated with each other, such as signal enhancement ratio in the MR imaging with the ${ }^{18} \mathrm{~F}$-FDG tissue uptake in the PET scan [25].

For the MR examination of breasts special coils are used, which are within the field-of-view of PET. They cause the scattering and attenuation of the photons produced in the reaction of annihilation, thus affecting the PET image. To improve such images, three-dimensional attenuation maps have been suggested [26]. Despite the advantages of MR and PET, PET/MR mammography has a similar sensitivity and specificity to MR mammography [27].

\section{New radiopharmaceuticals}

Due to the abovementioned limitations of ${ }^{18} \mathrm{~F}-\mathrm{FDG}$, there have been a number of studies investigating new tracers among breast cancer patients. One of them is an analogue of estradiol, $16 \alpha-{ }^{18} \mathrm{~F}$-fluoro-17 $\beta$-estradiol (FES). PET/CT with FES allows the assessment of ER expression, while being non-invasive and reproducible. This has a profound clinical impact because the ER expression may change after the onset of the disease and during treatment. Moreover, it can differ between the primary tumour and the metastases. In certain centres FES PET/CT is used when biopsy is not possible or when it has yielded ambiguous results [28]. The study of van Krutchen et al. showed that $45 \%$ of patients had both ER+ and ER- metastases, and the FES PET/CT scan resulted in a change of treatment among 48\% of them [28]. The assessment of ER expression using FES is only difficult in liver metastases due to the high physiological estradiol uptake by this organ [28]. Oestrogen analogues (such as tamoxifen) may inhibit FES uptake. This is why such medications have to be suspended for a longer period of time before the FES PET/CT examination. Premenopausal estradiol levels seem to have no influence on FES uptake, whereas the levels of sex hormone-binding globulin (SHBG) seem to exert a certain influence on the cellular uptake of FES [29].

Another tracer that has entered clinical practice is an analogue of thymidine, $\left[{ }^{18} \mathrm{~F}\right]$ 3'-deoxy-3'-fluorothymidine (FLT). Its uptake is correlated with the activity of thymidine kinase, and thus the proliferative activity of cells, which can be useful, e.g. in monitoring response to docetaxel treatment [30].

In conclusion, PET and PET/CT allow us to examine breast cancer patients thoroughly in one scan - both in terms of anatomy and cellular metabolism. They have been shown to influence the management in all stages of the disease, both among patients with primary breast cancer and in suspected recurrences. Moreover, they are tools that make it possible to detect early response to treatment, and thus help in choosing the most effective therapy. The fusion of PET and MR imaging and the development of new radiotracers present even wider perspectives for PET imaging in oncology. It seems likely that in the near future, in spite of relatively high costs, a variety of PET-based techniques will become part of routine clinical practice.

The authors declare no conflict of interest.

\section{References}

1. Adams HJ, Nievelstein RA, Kwee TC. Prognostic value of interim FDG-PET in Hodgkin lymphoma: systematic review and meta-analysis. Br J Haematol 2015; 170: 356-66.

2. Jeong JU, Chung WK, Nam TK, et al. Early metabolic response on 18F-fluorodeoxyglucose-positron-emission tomography/computed tomography after concurrent chemoradiotherapy for advanced stage III non-small cell lung cancer is correlated with local tumor control and survival. Anticancer Res 2014; 34: 2517-23.

3. Blom RL, Steenbakkers IR, Lammering G, et al. PET/CT-based metabolic tumour volume for response prediction of neoadjuvant chemoradiotherapy in oesophageal carcinoma. Eur J Nucl Med Mol Imaging 2013; 40: 1500-6.

4. Cheng X, Li Y, Liu B, Xu Z, Bao L, Wang J. 18F-FDG PET/CT and PET for evaluation of pathological response to neoadjuvant chemotherapy in breast cancer: a meta-analysis. Acta Radiol 2012; 53: 615-27.

5. Mghanga FP, Lan X, Bakari KH, Li C, Zhang Y. Fluorine-18 fluorodeoxyglucose positron emission tomography-computed tomography in monitoring the response of breast cancer to neoadjuvant chemotherapy: a meta-analysis. Clin Breast Cancer 2013; 13: 271-9.

6. Wang Y, Zhang C, Liu J, Huang G. Is 18F-FDG PET accurate to predict neoadjuvant therapy response in breast cancer? A meta-analysis. Breast Cancer Res Treat 2012; 131: 357-69.

7. Kolesnikov-Gauthier H, Vanlemmens L, Baranzelli MC, Vennin P, Servent V, Fournier C, Carpentier P, Bonneterre J. Predictive value of neoadjuvant chemotherapy failure in breast cancer using FDG-PET after the first course. Breast Cancer Res Treat 2012; 131: 517-25.

8. Koolen BB, Pengel KE, Wesseling J, et al. FDG PET/CT during neoadjuvant chemotherapy may predict response in ER-positive/ HER2-negative and triple negative, but not in HER2-positive breast cancer. Breast 2013; 22: 691-7.

9. Groheux D, Hatt M, Hindié E, et al. Estrogen receptor-positive/ human epidermal growth factor receptor 2-negative breast tumors: early prediction of chemosensitivity with (18)F-fluorodeoxyglucose positron emission tomography/computed tomography during neoadjuvant chemotherapy. Cancer 2013; 119: 1960-8.

10. Zucchini G, Quercia S, Zamagni C, Santini D, Taffurelli M, Fanti S, Martoni AA. Potential utility of early metabolic response by $18 \mathrm{~F}-2$-fluoro-2-deoxy-D-glucose-positron emission tomography/computed tomography in a selected group of breast cancer patients receiving preoperative chemotherapy. Eur J Cancer 2013; 49: 1539-45.

11. Groheux D, Hindié E, Giacchetti S, et al. Triple-negative breast cancer: early assessment with 18F-FDG PET/CT during neoadjuvant chemotherapy identifies patients who are unlikely to achieve a pathologic complete response and are at a high risk of early relapse. J Nucl Med 2012; 53: 249-54.

12. Mortazavi-Jehanno N, Giraudet AL, Champion L, et al. Assessment of response to endocrine therapy using FDG PET/CT in metastatic breast cancer: a pilot study. Eur J Med Mol Imaging 2012; 39: 450-60.

13. Buchbender C, Kuemmel S, Hoffmann O, et al. FDG-PET/CT for the early prediction of histopathological complete response to neoadjuvant chemotherapy in breast cancer patients: initial results. Acta Radiol 2012; 53: 628-36. 
14. Huyge V, Garcia C, Alexiou J, et al. Heterogeneity of metabolic response to systemic therapy in metastatic breast cancer patients. Clin Oncol 2010; 22: 818-27.

15. Dirisamer A, Halpern BS, Flöry D, Wolf F, Beheshti M, Mayerhoefer $M E$, Langsteger $W$. Integrated contrast-enhanced diagnostic whole-body PET/CT as a first-line restaging modality in patients with suspected metastatic recurrence of breast cancer. Eur J Radiol 2010; 73: 294-9.

16. Murakami R, Kumita S, Yoshida T, et al. FDG-PET/CT in the diagnosis of recurrent breast cancer. Acta Radiol 2012; 53: 12-6.

17. Aukema TS, Rutgers EJ, Vogel WV, et al. The role of FDG PET/CT in patients with locoregional breast cancer recurrence: A comparison to conventional imaging techniques. Eur J Surg Oncol 2010; 36: 387-92.

18. Heusner TA, Freudenberg LS, Kuehl H, Hauth EA, Veit-Haibach P, Forsting M, Bockisch A, Antoch G. Whole-body PET/CT-mammography for staging breast cancer: initial results. Br J Radiol 2008; 81: 743-8.

19. Champion L, Brain E, Giraudet AL, et al. Breast cancer recurrence diagnosis suspected on tumor marker rising: value of whole-body 18FDG-PET/CT imaging and impact on patient management. Cancer 2011; 117: 1621-9.

20. lima M, Nakamoto Y, Kanao S, et al. Clinical performance of 2 dedicated PET scanners for breast imaging: initial evaluation. J Nucl Med 2012; 53: 1534-42.

21. Zytoon AA, Murakami K, El-Kholy MR, El-Shorbagy E. Dual time point FDG-PET/CT imaging. Potential tool for diagnosis of breast cancer. Clin Radiol 2008; 63: 1213-27.

22. Carkaci S, Macapinlac HA, Cristofanilli M, et al. Retrospective study of 18F-FDG PET/CT in the diagnosis ofinflammatory breast cancer: preliminary data. J Nucl Med 2009; 50: 231-8.

23. Hahn S, Hecktor J, Grabellus F, et al. Diagnostic accuracy of dual-time-point 18F-FDG PET/CT for the detection of axillary lymph node metastases in breast cancer patients. Acta Radiol 2012; 53: 518-23.

24. Moy L, Noz ME, Maguire GQ Jr, et al. Role of fusion of prone FDGPET and magnetic resonance imaging of the breasts in the evaluation of breast cancer. Breast J 2010; 16: 369-76.

25. Partridge SC, Vanantwerp RK, Doot RK, et al. Association between serial dynamic contrast-enhanced MRI and dynamic 18F-FDG PET measures in patients undergoing neoadjuvant chemotherapy for locally advanced breast cancer. J Magn Reson Imaging 2010; 32: 1124-31.

26. Aklan B, Paulus DH, Wenkel E, et al. Toward simultaneous PET/MR breast imaging: Systematic evaluation and integration of a radiofrequency breast coil. Med Phys 2013; 40: 024301/11.

27. Heusner TA, Hahn S, Jonkmanns C, et al. Diagnostic accuracy of fused positron emission tomography/magnetic resonance mammography: initial results. Br J Radiol 2011; 84: 126-35.

28. van Kruchten M, Glaudemans AW, de Vries EF, Beets-Tan RG, Schröder CP, Dierckx RA, de Vries EG, Hospers GA. PET Imaging of estrogen receptors as a diagnostic tool for breast cancer patients presenting with a clinical dilemma. J Nucl Med 2012; 53: 182-90.

29. Peterson LM, Kurland BF, Link JM, Schubert EK, Stekhova S, Linden HM, Mankoff DA. Factors influencing the uptake of 18F-fluoroestradiol in patients with estrogen receptor positive breast cancer. Nucl Med Biol 2011; 38: 969-78.

30. Contractor KB, Kenny LM, Stebbing J, et al. [18F]-30Deoxy-30-fluorothymidine positron emission tomography and breast cancer response to docetaxel. Clin Cancer Res 2011; 17: 7664-72.

\section{Address for correspondence}

Rafał Czepczyński, PhD

Department of Endocrinology and Metabolism

Poznan University of Medical Sciences

Poznan, Poland

e-mail: tarmed@poczta.onet.pl

Submitted: 22.12 .2014

Accepted: 20.07 .2015 